\title{
Random fiber lasers: application to fiber optic sensors networks
}

\author{
Manuel Lopez-Amo Senior Member, IEEE, Daniel Leandro, Verónica de Miguel, Mikel Bravo, \\ Montserrat Fernández-Vallejo and Rosa Ana Perez-Herrera \\ Universidad Publica de Navarra, Dept. of Electrical and Electronic Engineering and Institute of Smart Cities, \\ Campus de Arrosadia S/N, E-31006, Pamplona, Spain \\ Tel: (34)948169260, Fax: (34)948169720, e-mail: mla@unavarra.es
}

\begin{abstract}
Recently, random mirrors have been proposed as a method to create fiber laser cavities. This kind of cavity is based on cooperative Rayleigh scattering, which is generated along a fiber due to the material inhomogeneities presented in that fiber. In this work, basics of Random fiber lasers and different demonstrated lasing sensors systems for interrogating arrays of optical fiber sensors are shown. These systems use different kinds of amplification and cavities schemes and can interrogate optical fiber sensors located up to $225 \mathrm{~km}$ away.
\end{abstract}

Keywords: Random fiber laser, optical fiber sensors, multiplexing, remote sensing

\section{INTRODUCTION}

Random distributed feedback fiber lasers are nowadays the subject of intense theoretical and experimental studies. In 2009 [1], these lasers were shown as a good sensing platform. In 2010 they were presented as a new kind of lasers suitable to obtain single wavelength [2] and multiwalength laser sources [3]. These lasers utilize distributed cavities called random mirrors, based on cooperative Rayleigh scattering (RS). Unlike conventional cavity fiber lasers, based on point reflectors or fiber optic loops, their principle of operation relies on distributed scattering events along the fiber cavity.

An example of a Random DFB fiber laser setup can be seen in Fig. 1, wherein single mode fiber is used to generate RS feedback in a double-arm topology. It should be noted that the optical fiber can act as both gain medium for the amplification and RS generator. In the case of using Raman amplification (the most common), several kilometers of SMF are typically used as active medium and distributed reflector simultaneously [2].

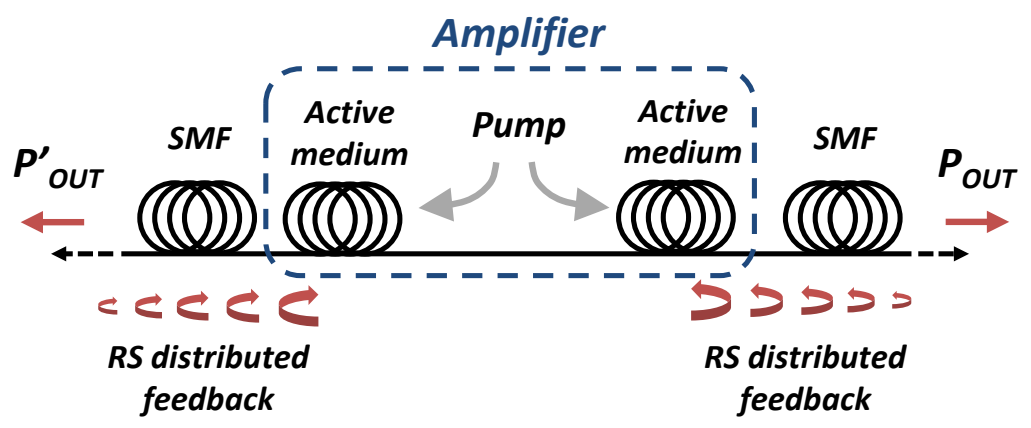

Figure 1. Basic scheme of a random distributed feedback fiber laser

The Rayleigh scattering in optical fibers comes from light interaction with particles much smaller than the wavelength of the radiation. It is a linear effect by which the incoming light interacts with the random density fluctuations created in the fiber during the manufacturing process. In this manner, a random distributed feedback cavity can be considered as a combination of a vast number of linear cavities with different fiber lengths and extremely weak reflectors. A random distributed feedback fiber laser has efficiency and performance that are comparable to and even exceed those of similar conventional fiber lasers. A key feature of the generated radiation of random distributed feedback fiber lasers include a very stable narrow-band continuous modeless spectrum that is free of mode competition. Thus, this lasers are particularly suitable for optical fiber sensors interrogation, and because the amplification process and the random mirror can be generated along tens of kilometers, they are also suitable for remote multiplexing of sensors.

\section{RANDOM FIBER LASERS FOR SENSING}

First random fiber laser (RFL) based on cooperative Rayleigh scattering for sensing was presented in [1]. In that work, a Raman based fiber Bragg grating (FBG) laser sensor for strain and temperature measurement was presented. The laser resonator consisted of a linear cavity formed by the reflectivity of the Bragg gratings and a 
virtual distributed mirror formed in a dispersion-compensating fiber, created by the Raman effect. Using one pump laser was possible to obtain a laser sensor with a range of $\sim 35 \mathrm{~nm}$. The sensing system was demonstrated when the fiber Bragg gratings were remotely located at a $10 \mathrm{~km}$ distance (see Fig. 1).

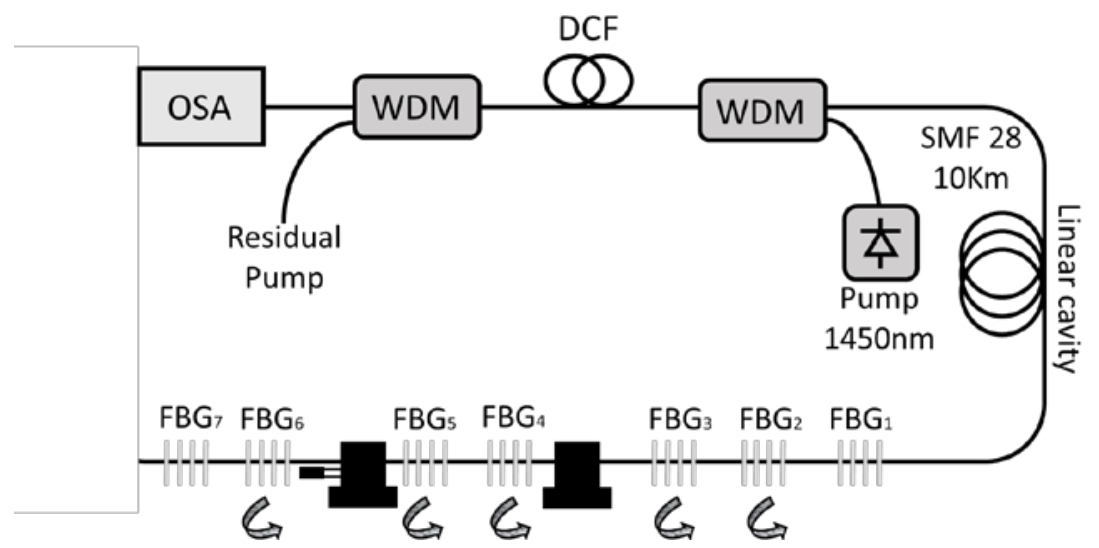

Figure 2. Set-up of the sensing system based on a random distributed feedback fiber laser demonstrated in [1]

Afterwards, several sensing systems based in RFL were proposed [4], showing good resolution and stabilities. For instance, a temperature sensor based on lasing provided by the combination of a Fabry-Pérot cavity and a RFL was proposed in [5].The Fabry-Pérot cavity, fabricated by the splicing of a single mode fiber with a small piece of suspended-core fiber was used simultaneously as a laser reflective mirror and a temperature sensing element.

More recently, in [6], high-resolution temperature measurements using a RFL were attained. A phase-shifted fiber Bragg grating was used to detect temperature shifts with a resolution under $0.01^{\circ} \mathrm{C}$.

In [7], two continuous-wave configurations, with single and cascaded cavities using fiber Bragg gratings as mirrors were explored for a $300 \mathrm{~km}$ long fiber. For optical sensing, the cavity length was optimized for $250 \mathrm{~km}$ using one of the gratings an intensity sensor. A FBG sensor placed as far as $300 \mathrm{~km}$ apart was interrogated in a two-wavelength random DFB fiber laser configuration: the scheme comprised a fiber loop mirror at one fiber end and the FBG-based sensor at the other end of the $300 \mathrm{~km}$ fiber span.

\section{REMOTE SENSING SYSTEMS USING RANDOM FIBER LASERS}

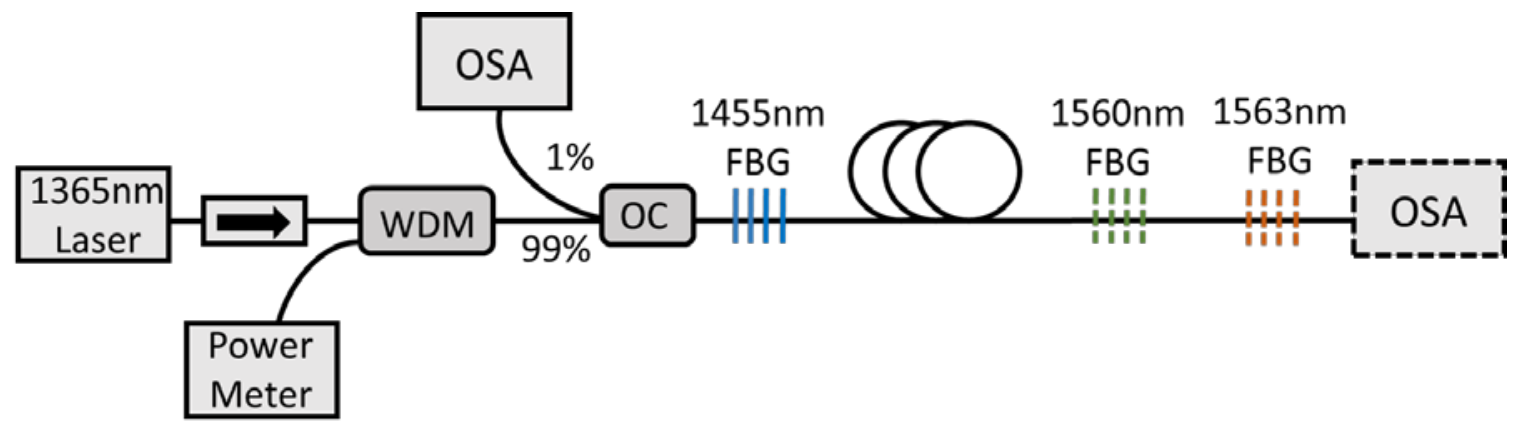

Figure 3. Remote sensing utilizing the second-order random lasing (Adapted from [8])

Remote fiber sensor systems could also benefit when random DFB fiber lasers are used. The main idea behind remote sensing concept is the continuous monitoring of sensors or structures from a central station located tens or hundreds of kilometers away from the field through the critical location of sensors, which send information to the central station, without the necessity of electrical power feeds in the remote locations. In this sense [7], cannot be considered as a remote sensing application, since has active elements at both ends of the structure. However, reference [1] could be included here as the first remote sensing system based on a RFL. More recently, in 2012, two $100 \mathrm{~km}$ fiber Bragg grating (FBG) point-sensing schemes based on RFLs were experimentally demonstrated using the first order and the second-order random lasing, respectively[8]. The system is shown in Figure 3. In 2013, a random fiber laser with a reach of $200 \mathrm{~km}$ was demonstrated to interrogate 11 FBG sensors at the end of the cavity [9]. In [10], the internal modulation of the generated power in random DFB fiber laser was demonstrated. This features RFL as a source that can be easily modulated for remote sensing or telecommunications, as demonstrated in [11]. There, the modulation of the laser's cavity allowed the sensors to 
be identified by means of time-domain reflectometry. Moreover, the reflected signal vs distance profile were measured at different wavelengths by tuning the emission wavelength of the laser. Thus, that setup overcomes one of the main drawbacks of ultra-long range remote sensing systems, that is their very limited multiplexing capability. With that multiplexing strategy, sensors located at the same wavelength can be identified by their position in the network. In the same manner, sensors closely spaced can be wavelength-multiplexed. The multiplexing network, shown in Figure 4.b, interrogated sensors located up to $200 \mathrm{~km}$ apart from the head of the network (Fig. 4.a).

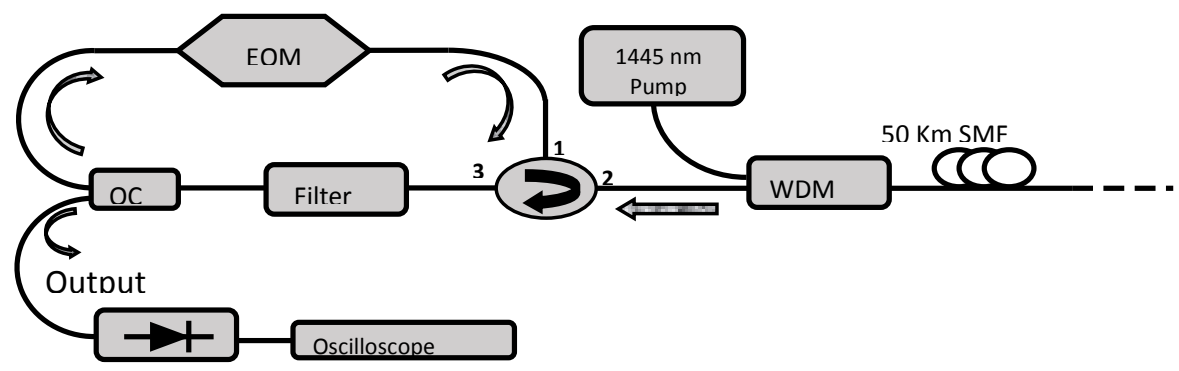

a)

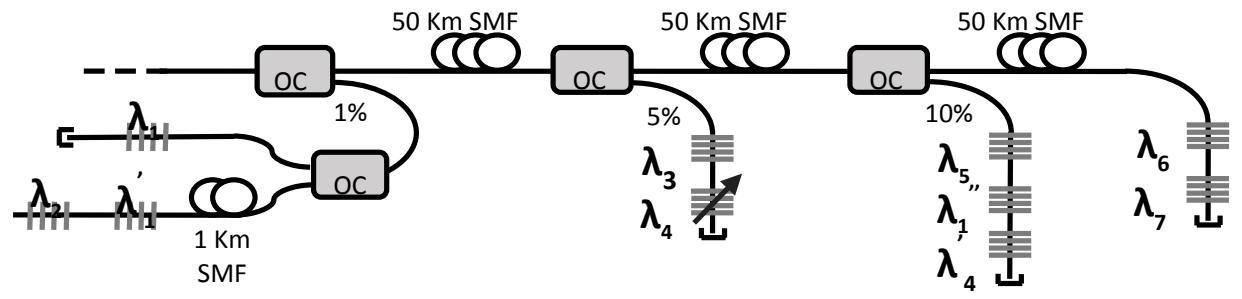

b)

Figure 4. Hybrid TDM/WDM 200 km long multiplexing network based on a Ramdom laser. a) Head of the network. b) Remotely interrogated FBG sensors. OC. Optical coupler, EOM: Electrooptical modulator, WDM: wavelength division multiplexer (Adapted from [11])
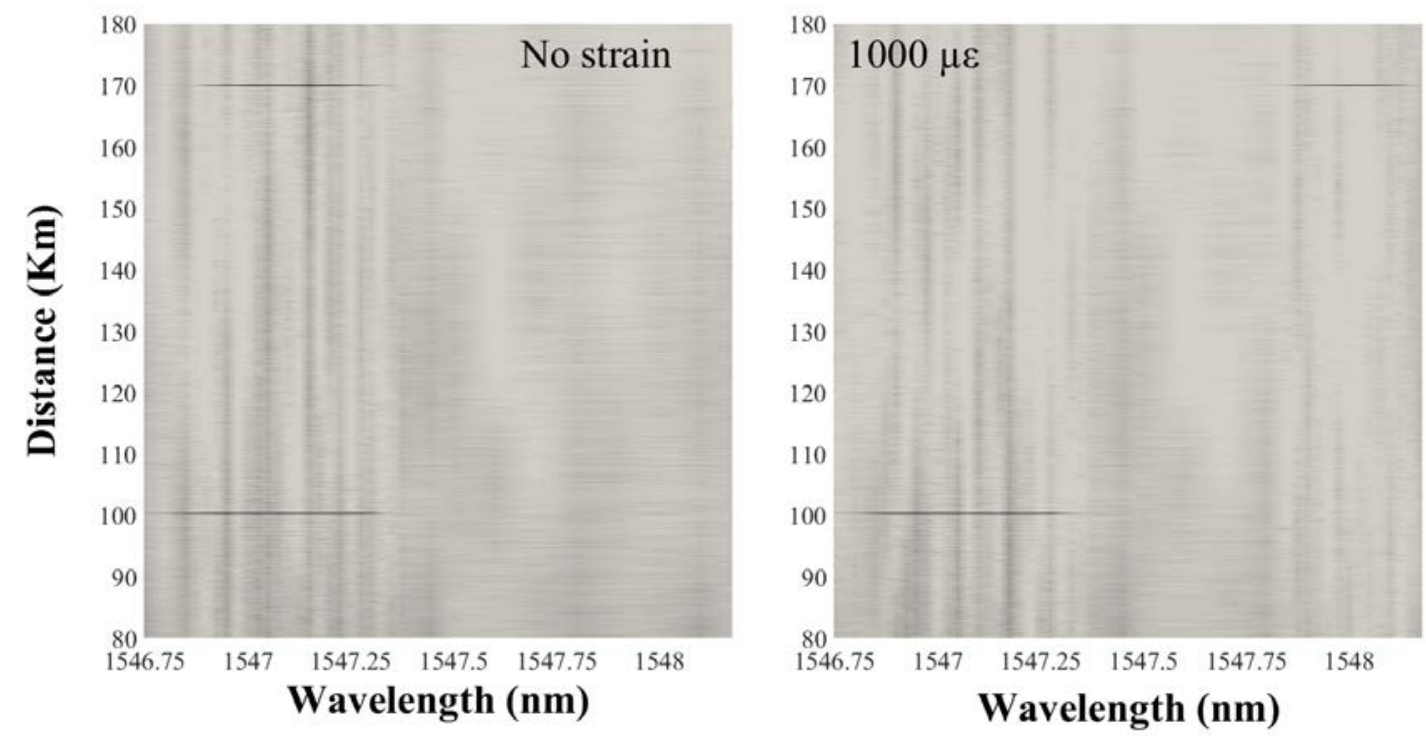

Figure 5. Strain measurements of a FBG sensor placed at $170 \mathrm{~km}$ in a random laser network as shown in [11] 
Recently [12], the use of the signal generated by a commercial fiber Bragg grating sensors interrogator as the seed of a random laser allowed monitoring the strain applied to a HiBi FLM interferometer, located 225 kilometers away from the monitoring station.

\section{CONCLUSIONS}

Random distributed feedback fiber lasers has been demonstrated as a new kind of lasers having a very stable narrow-band continuous modeless spectrum that is free of mode competition. This stable optical radiation makes random lasers well suited for optical fiber sensors interrogation, and because the amplification process and the random mirror can be generated along tens of kilometers, they are also suitable for remote multiplexing of sensors. Several examples of random laser based sensing systems has been reviewed in this paper. Also, their utilization as a remote sensing platform has been reviewed, showing demonstrations of systems that can interrogate sensors placed up to $225 \mathrm{~km}$ away from the interrogation unit.

\section{ACKNOWLEDGEMENTS}

The authors would like to thank the financial support from Spanish Comisión Interministerial de Ciencia y Tecnología within projects TEC2016-76021-C2-1-R and TEC2013-47264-C2-2-R, Campus Iberus and FEDER funds from the European Union.

\section{REFERENCES}

[1] O. Frazão, C. Correia, J.L. Santos and J.M. Baptista: Raman fibre Bragg-grating laser sensor with cooperative Rayleigh scattering for strain-temperature measurement, Meas. Sci. Technol. Vol. 20 pp. 045203 (5pp), 2009.

[2] S. K. Turitsyn, S. A. Babin, A. E. El-Taher, P. Harper, D. V. Churkin, S. I. Kablukov, J. D. AniaCastanon, V. Karalekas and E. V. Podivilov: Random distributed feedback fiber laser, Nature Photonics, vol.4, pp. 231-235, 2010.

[3] A.M.R. Pinto, O. Frazão, J.L. Santos, M. López-Amo: Multiwavelength fiber laser based on a photonic crystal fiber loop mirror with cooperative Rayleigh scattering, Appl. Physics B, Vol 3, pp 391-395, 2010.

[4] S.K. Turitsyn, S.A. Babin, D.V. Churkin, I.D. Vatnik, M. Nikulin, E.V. Podivilov: Random distributed feedback fibre lasers, Physics Reports Vol. 542 (2), pp. 133-193, 2014.

[5] A.M.R. Pinto, M. Lopez-Amo, J. Kobelke, K. Schuster: Temperature fiber laser sensor based on a hybrid cavity and a random mirror, J. Lightwave Technol. Vol.30 (8) pp. 1168-1172, 2012.

[6] D. Leandro, V. deMiguel-Soto and M. Lopez-Amo: High-Resolution Sensor System Using a Random Distributed Feedback Fiber Laser, J. of Lightwave Tech Vol. 34, pp. 4596- 4602, 2016.

[7] H. Martins, M.B. Marques, O. Frazão, 300 km-ultralong Raman fiber lasers using a distributed mirror for sensing applications, Opt. Express 19 (19) pp.18149, 2011.

[8] Z.N. Wang, Y.J. Rao, H. Wu, P.Y. Li, Y. Jiang, X.H. Jia, W.L. Zhang: Long-distance fiber-optic pointsensing systems based on random fiber lasers, Opt. Express Vol. 20 (16) pp. 17695-17700, 2012.

[9] M. Fernandez-Vallejo, M. Bravo, M. Lopez-Amo: Ultra-long laser systems for remote fiber Bragg gratings arrays interrogation, IEEE Photon. Technol. Lett. Vol. 25 (14) pp. 1362-1364, 2013.

[10] M. Bravo, M. Fernandez-Vallejo, and M. Lopez-Amo, "Internal modulation of a random fiber laser," Opt. Lett., vol. 38 (9) pp. 1542-1544, 2013.

[11] D. Leandro, V. deMiguel Soto, R.A. Perez-Herrera, M. Bravo and M. Lopez-Amo: Random DFB Fiber Laser for Remote (200 km) Sensor Monitoring Using Hybrid WDM/TDM J. of Lightwave Tech., Vol. 34 (19), 2016.

[12] V. DeMiguel-Soto, A. Lopez-Aldaba, D. Leandro, M. Lopez-Amo: Ultra-Long random laser for remote real-time interferometric sensor monitoring using FFT analysis, In Proc APOS 2016, Shanghai, China Oct. 2106, paper Tu3A.4. 